\title{
Mass determination and event reconstruction at Large Hadron Collider
}

\author{
Abhaya Kumar Swain and Partha Konar
}

\begin{abstract}
After successful discovery of the Higgs boson, the Large Hadron Collider (LHC) would confront the major challenge in searching for new physics and new particles. Any such observation necessitates the determination of mass and other quantum numbers like spin, polarisation etc. Many of our theories beyond the Standard Model (BSM) motivated from profound experimental indication of dark matter (DM), trying to accommodate them as some stable BSM particles within these theory. In such scenario, any production of heavy resonance of new particles eventually decay semi-invisibly resulting at least two stable particles in the final state. Reconstruction of these events at hadron colliders together with the mass determination of DM or intermediate particles is challenging and center to this present analysis. In this work we discuss some mass restricting way that can lead us to determine the new particle mass when it decays semi-invisibly. We will also present a new method which can be used for the full reconstruction of the event in the above scenario.
\end{abstract}

\section{Introduction}

The remarkable discovery of Higgs boson by ATLAS [1] and CMS [2] at LHC has resolved a long standing question in the Standard Model (SM). Apart from acquiring more Higgs data to significantly improve the measurements, the prime goal of Large Hadron Collider (LHC) is to search for the physics beyond Standard Model (BSM). Buoyed by significant number of experimental evidence on dark matter, most of the BSM theories accommodate dark matter (DM) as some new stable fun-

Abhaya Kumar Swain

Theoretical Physics Division, Physical Research Laboratory, Ahmedabad 380009, India, e-mail: abhayaeprl.res.in

Partha Konar

Theoretical Physics Division, Physical Research Laboratory, Ahmedabad 380009, India, e-mail: konareprl.res.in 
damental particle. Detection of these DM particles in LHC is challenging because it goes missing in the detector and only manifest itself through missing energy signal. Additional complexity is introduced by the nature of hadron collider from the availability of partial informations of incoming parton momenta. Lot of efforts were gone to deal with missing transverse energy signals and to determine the quantities like mass, spin and polarisation of particles associated with those signals. For a recent review one can follow from ref. [3, 4]. Among all the inclusive and global mass determination variables $\hat{s}_{\min }$ and its variants [5, 6] are quiet interesting as they provide the mass scale of new physics without worrying about the production mechanism. These variables can also handle any number of invisibles and variety of them and simple analytical formula is also exists.

In this paper we try to formulate the usefulness of the topology information in the inclusive variable $\hat{s}_{\min }$ by implementing the on shell constraints in the minimization. Principal consequence being the constrained phase space which is bounded from below, as well as from above. So we define two new variables $\hat{s}_{\min }^{c o n s}$ and $\hat{s}_{\max }^{c o n s}$ which can be used as mass constraining variables. We also showed that $\hat{s}_{\min }$ can be used for a unique event reconstruction for any topology and the momentum reconstruction can be improved significantly by these constrained variables. This paper is organized as follows: we will give a very brief introduction about the variable $\hat{s}_{\min }$ and the constrained variables in section 2 . We will discuss the event reconstruction capability of these variables in section 3 followed by conclusion at section 4.

\section{$2 \hat{s}_{\min }$ variable and its constrained counterpart using topology information}

The partonic mandelstam variable $\hat{s}$ contains information like mass of heavy resonance for Antler topology or threshold of pair production of particles for non-Antler topology. Given a generic topology the system is under constrained, where it is nearly impossible to determine $\hat{s}$ experimentally which is the primary motivation to construct the variable $\hat{s}_{\min }$ by minizing $\hat{s}$ w.r.t unknown invisible momenta subject to missing energy constraint. In other words $\hat{s}_{\min }$ is the minimum partonic mandelstam variable that is consistent with all the visible particle momenta in the final state and missing transverse momentum constraints. It is a global and inclusive variable which makes it applicable to apply any topology with a simple analytical formula.

We simplify our discussion by making two assumptions which are common in BSM theories, that the dark matter stabilizing symmetry is a $Z_{2}$ symmetry and there is only one dark matter candidate in the theory under consideration. In this draft we restrict ourself for symmetric Antler topology as given in the figure 1 where $\mathrm{G}$ is a $Z_{2}$ parity even heavy resonance decays to two parity odd resonance $M_{1}$ and $M_{2}$ each of which further decays to two SM particles $V_{k}$ and one DM particles $I_{i}$. The four momenta of visible and invisible particles are $p_{k}$ and $q_{i}$ respectively. The analytical formula for $\hat{s}_{\min }$ and invisible momentum at the minimum for symmetric Antler topology can be found in the ref [5]. The on shell constraints which $\hat{s}_{\min }$ have 

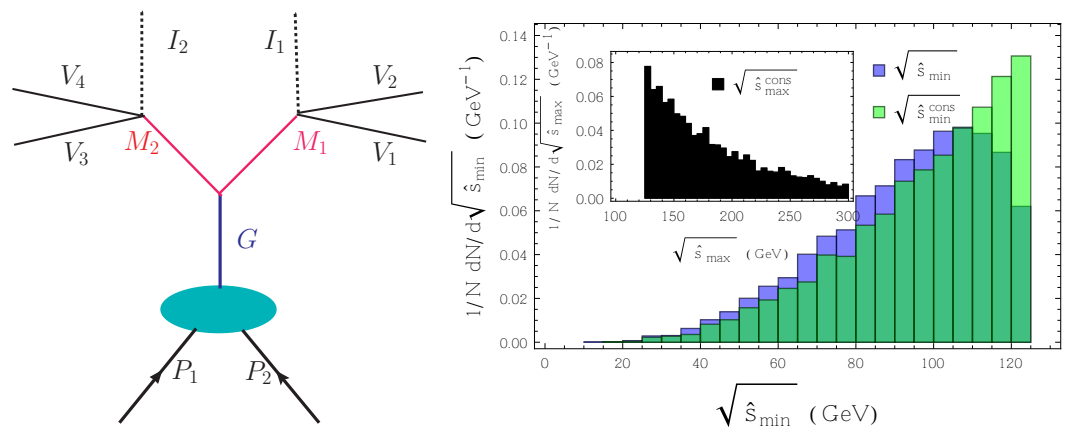

Fig. 1 The diagram on left represents Antler topology where $\mathrm{G}$ is a parity even resonance decays to two parity odd resonance $M_{1}$ and $M_{2}$ each of which further decays to two SM particles $V_{k}, k=$ $1,2,3,4$ and one DM particle $I_{i}, i=1,2$. The right side figure shows distribution of $\hat{s}_{\min }$ in blue, $\hat{s}_{\min }^{\text {cons }}$ in green and the black figure inside it represents $\hat{s}_{\max }^{\text {cons }}$.

not used in its minimization is the mass shell constraints of $M_{1}$ and $M_{2}$, we assume we know the masses 1 of these two resonance and use them to constrain the phase space. We referred to constraints as

$$
\text { constraints }=\left\{\begin{array}{l}
\left(p_{1}+p_{2}+q_{1}\right)^{2}=M_{M_{1}}^{2},\left(p_{3}+p_{4}+q_{2}\right)^{2}=M_{M_{2}}^{2} \\
q_{1}^{2}=M_{I_{1}}^{2}, q_{2}^{2}=M_{I_{2}}^{2} \\
\mathbf{q}_{1 T}+\mathbf{q}_{2 T}=\mathbf{P}_{T} .
\end{array}\right\}
$$

$\left\{M_{M_{1}}, M_{M_{2}}\right\}$ and $\left\{M_{I_{1}}, M_{I_{2}}\right\}$ are the true masses of the intermediate particles $\left\{M_{1}\right.$, $\left.M_{2}\right\}$ and the invisible particles $\left\{I_{1}, I_{2}\right\}$ respectively. Now we define constrained variables using the equations in constraints as

$$
\begin{aligned}
& \hat{s}_{\text {min }}^{\text {cons }}=\min _{\substack{\mathbf{q}_{1} \mathbf{q}_{\mathbf{2}} \\
\{\text { constraints }\}}}\left[\hat{s}\left(\mathbf{q}_{1}, \mathbf{q}_{\mathbf{2}}\right)\right] \\
& \hat{s}_{\text {max }}^{\text {cons }}=\max _{\substack{\mathbf{q}_{1} \mathbf{q}_{2} \\
\{\text { constraints }\}}}\left[\hat{s}\left(\mathbf{q}_{1}, \mathbf{q}_{\mathbf{2}}\right)\right]
\end{aligned}
$$

$\hat{s}_{\min }^{\text {cons }}$ is defined as the minimum of the constrained phase space which is bounded above by true of G. $\hat{s}_{\min }^{\text {cons }}$ being the minimum of the constrained phase space it can not exceed the true mass of $\mathrm{G}$, if it does that will point out that the constrained phase space does not contain the true mass point. $\hat{s}_{\max }^{\text {cons }}$ defined as the maximum of the constrained phase space which bounded below true mass of $\mathrm{G}$ because of the same reason given for $\hat{s}_{\min }^{c o n s}$. The details about these variables can be found the ref. [7]. The distribution of these variables with $\hat{s}_{\min }$ is given in the figure 1 ; blue histogram

${ }^{1}$ There are many examples in SM and beyond Standard Model theories as given in the ref. [7] where we know the intermediate particles mass. 

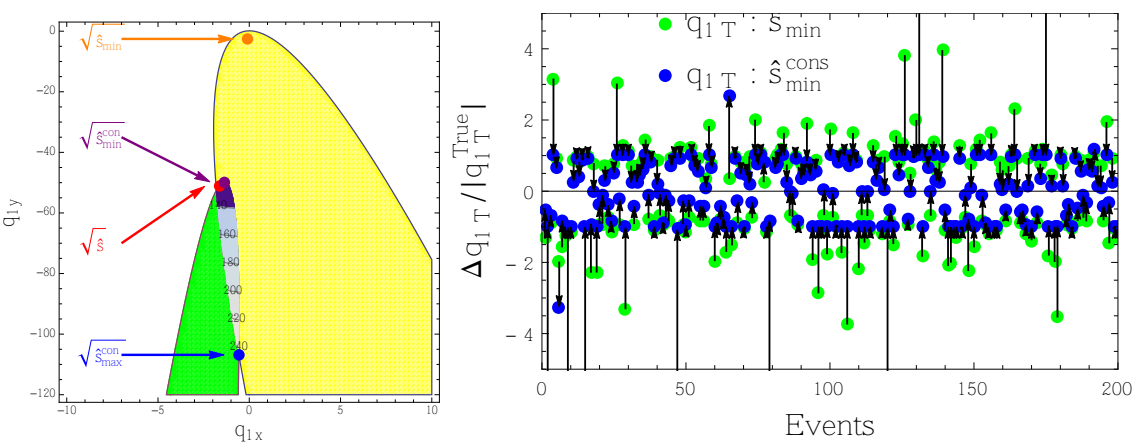

Fig. 2 Figure on left shows the constraint phase space coming from the intersection of the two ellipses using the on shell constraints for one event. $\hat{s}_{\min }, \hat{s}_{\min }^{c o n s}, \hat{s}_{\max }^{c o n s}$ and $\hat{s}^{T r u e}$ are presented by orange, purple, blue and red points respectively. As can be seen the constrained variables performing better for this event. Figure on right shows for 200 events the improvement of constrained variable over unconstrained one and also well correlated with true momenta.

represents $\hat{s}_{\text {min }}$, green shows $\hat{s}_{\text {min }}^{\text {cons }}$ and the black histogram inside this figure is for $\hat{s}_{\max }^{\text {cons }}$.

\section{3 event reconstruction capability}

In this section we describe the momentum reconstruction capability of $\hat{s}_{\text {min }}$ and its constrained counterpart $\hat{s}_{\text {min }}^{\text {cons }}$. One can also assign momenta to invisible particles using $\hat{s}_{\text {max }}^{\text {cons }}$, which can be found in the ref. [7]. In this paper we assign that momenta to invisible particles which gives $\hat{s}_{\text {min }}$ or $\hat{s}_{\min }^{\text {cons }}$ that is the momenta from the minimization. Now using the two assumptions described in section 2 the momenta that gives $\hat{s}_{\text {min }}$ are,

$$
\begin{aligned}
q_{i T} & =\frac{1}{2} \mathbf{P}_{T}, \\
q_{i z} & =\frac{1}{2} \frac{\mathbf{P}_{T}}{\sqrt{\left(E^{v}\right)^{2}-\left(P_{z}^{v}\right)^{2}}} \sqrt{M_{i n v}^{2}+\mathbf{P}_{T}^{2}} .
\end{aligned}
$$

Where $\mathbf{P}_{T}, E^{v}, P_{z}^{v}$ and $M_{i n v}$ are missing transverse momenta, total visible energy, total visible longitudinal momenta and sum of invisible particle masses respectively. We improve this momentum reconstruction by using on shell constraints of $B_{1}$ and $B_{2}$ in the minimization. The two on shell constraints of $B_{1}$ and $B_{2}$ are two ellipses and they are related by the missing energy constraints. So the intersection region satisfies all the six constraints (4 mass shell constraints +2 missing energy constraints) and the minimum of that constrained phase space is called $\hat{s}_{\min }^{\text {cons }}$ and maximum is called $\hat{s}_{\text {max }}^{\text {cons }}$ which is shown in the left plot of Fig. 2. In the right figure we have shown correlation between reconstructed momenta and true momenta for 200 
events, where the green point shows the momenta from $\hat{s}_{\min }$, blue point shows momenta from $\hat{s}_{\min }^{\text {cons }}$ and black arrow points the motion of these points for each event.

\section{Conclusion}

- $\hat{s}_{\min }$ is a global and inclusive variable defined to measure the mass scale of new physics and it only depends on the momenta of final state visible particles and missing transverse energy. The simple analytical formula of $\hat{s}_{\min }$ allows one to calculate the mass scale of new physics without worrying about the number of invisible and variety of them in the topology.

- In this paper we made two simplifying assumptions which are mostly common in BSM theories: The DM stabilization symmetry is a $Z_{2}$ symmetry resulting at least two invisible particle in the final state, there is only one DM candidate in the theory. Now using these two assumptions one can approximate the unknown invisible momenta with the unique description from the minimization $\hat{s}_{\min }$.

- We added the topology information and partial mass spectrum information in $\hat{s}_{\min }$ if we already have some of them. These additional input constraints the allowed phase space further. This system is such bounded, the minimum of constrained region is dubbed as $\hat{s}_{\min }^{\text {cons }}$, bounded above by the true mass of G. Where as, the maximum of constrained region is dubbed as $\hat{s}_{\max }^{\text {cons }}$, bounded below by the mass of G. The relations between all such variables together with true mass are $\hat{s}_{\min } \leq$ $\hat{s}_{\min }^{\text {cons }} \leq M_{G} \leq \hat{s}_{\max }^{\text {cons }}$.

- The constrained variables can also be used for event reconstruction and a comparison has been made between momentum reconstruction capability of constrained and unconstrained variables. Both $\hat{s}_{\min }^{c o n s}$ significantly improves momentum reconstruction of invisibles giving better estimate for events near the endpoint, whereas, $\hat{s}_{\max }^{c o n s}$ gives better momentum reconstruction for events near threshold.

\section{References}

1. G. Aad et al. [ATLAS Collaboration], Phys. Lett. B 716, 1 (2012) [arXiv:1207.7214 [hepex]].

2. S. Chatrchyan et al. [CMS Collaboration], Phys. Lett. B 716, 30 (2012) [arXiv:1207.7235 [hep-ex]].

3. A. J. Barr and C. G. Lester, J. Phys. G 37, 123001 (2010) [arXiv:1004.2732 [hep-ph]].

4. A. J. Barr, T. J. Khoo, P. Konar, K. Kong, C. G. Lester, K. T. Matchev and M. Park, Phys. Rev. D 84, 095031 (2011) [arXiv:1105.2977 [hep-ph]].

5. P. Konar, K. Kong and K. T. Matchev, JHEP 0903, 085 (2009) [arXiv:0812.1042 [hep-ph]].

6. P. Konar, K. Kong, K. T. Matchev and M. Park, JHEP 1106, 041 (2011) [arXiv:1006.0653 [hep-ph]].

7. A. K. Swain and P. Konar, JHEP 1503, 142 (2015) [arXiv:1412.6624 [hep-ph]]. 